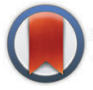

CrossMark \&lick for updates

Cite this: Phys. Chem. Chem. Phys., 2016, 18, 10289

Received 10th November 2015, Accepted 21st March 2016

DOI: $10.1039 / \mathrm{c} 5 \mathrm{cp} 06866 \mathrm{e}$

www.rsc.org/pccp

\title{
The ultrafast reactions in the photochromic cycle of water-soluble fulgimide photoswitches $\uparrow$
}

\author{
C. Slavov, ${ }^{a}$ C. Boumrifak, ${ }^{a}$ C. A. Hammer, ${ }^{a}$ P. Trojanowski, ${ }^{a}$ X. Chen, ${ }^{b}$ W. J. Lees, ${ }^{b}$ \\ J. Wachtveitl ${ }^{a}$ and M. Braun*a
}

\begin{abstract}
Photochromic switches are essential for the control and manipulation of nanoscale reactions and processes. The expansion of their application to aqueous environments depends strongly on the development of optimized water-soluble photoswitches. Here we present a femtosecond time-resolved investigation of the photochromic reactions (transition between the open and the closed form) of a water-soluble indolylfulgimide. We observe a pronounced effect of the protic nature of water as a solvent on the ultrafast ring-opening reaction. Typically, the excited state of the closed form has a larger dipole moment than the ground state, which leads to stabilization of the excited state in polar solvents and hence a lifetime (3 ps) longer than in non-polar solvents ( 2 ps). However, in water, despite the increased solvent polarity and the increased excited state dipole moment, the opposite trend for the excited state lifetime is observed (1.8 ps). This effect is caused by the opening of a new excited state deactivation pathway involving proton transfer reactions.
\end{abstract}

\section{Introduction}

Contemporary (bio)chemical science and (bio)technology are focused on the understanding, control and utilization of nanoscale reactions and structures, which necessitates the development of specialized manipulation tools. One promising technique uses molecular photochromic switches (photoswitches). Photoswitches are capable of reversible, lightinduced transition between forms with different physicochemical properties. ${ }^{1,2}$ The utilization of light is advantageous since it permits instantaneous, precise and most importantly noninvasive manipulation.

There are several classes of photochromic switches (e.g. azo compounds, diarylethenes, fulgides, spiropyrans, etc.) that are relatively well characterized and actively applied in research and industry. ${ }^{3-8}$ However, most photochromic compounds have low solubility and stability in water and thus their application is restricted to non-aqueous environments. To date, there is a limited number of studies of photoswitches in aqueous solutions, ${ }^{9-17}$ of which only a few are focused on the investigation of the ultrafast photochemical reactions (e.g. spiropyran ${ }^{13}$ and azobenzene ${ }^{18}$ ). Thus, despite the importance of water as a

\footnotetext{
${ }^{a}$ Institute of Physical and Theoretical Chemistry, Goethe University, 60438 Frankfurt/Main, Germany. E-mail: braun@theochem.uni-frankfurt.de ${ }^{b}$ Biomolecular Sciences Institute, School of Integrated Sciences and Humanities and Department of Chemistry and Bio-chemistry, Florida International University, Miami, Florida 33199, USA

$\dagger$ Electronic supplementary information (ESI) available: Supplementary figures. See DOI: $10.1039 / \mathrm{c} 5 \mathrm{cp} 06866 \mathrm{e}$
}

solvent environment for chemical and biological processes and the excessive demand for their control and manipulation, the behaviour of photoswitches in aqueous surroundings remains largely uncharacterized. This deficiency represents a major obstacle to a more extensive utilization of photoswitches.

Fulgides are photochromic switches derived from aryl substituted dimethylenesuccinic anhydride (Scheme 1). The substitution leads to the formation of a 1,3,5-hexatriene motif, which permits $6 \pi$-electrocyclization to cyclohexadiene. Therefore, these photoswitches exist in three isomeric forms - open $Z$ - and $E$-forms (colourless hexatriene motif) and closed $C$-form (coloured cyclohexadiene motif). ${ }^{6}$ The $Z$ - and the $E$-forms have similar optical properties and can be interconverted using near UV light. ${ }^{19}$ However, the photochromic reaction of interest with the strongest change of optical properties involves the electrocyclic ring-closing of the cyclizable open form to the thermally stable $C$-form (Fig. 1). ${ }^{6}$ Of particular interest are indolylfulgides, which have a bulkier aryl substituent and thus exhibit a reduced formation of the non-cyclizable open form. Furthermore, they

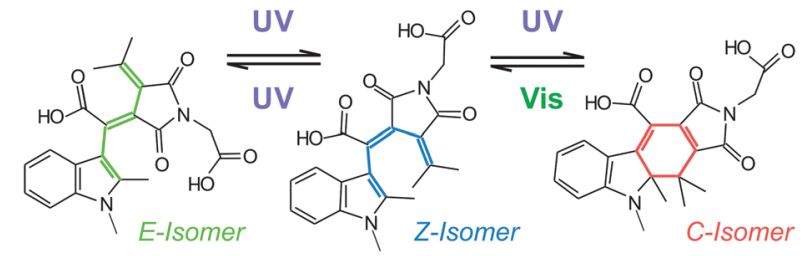

Scheme 1 Chemical structures of WF (closed form (C); non-cyclizable open form (E); cyclizable open form (Z)). 


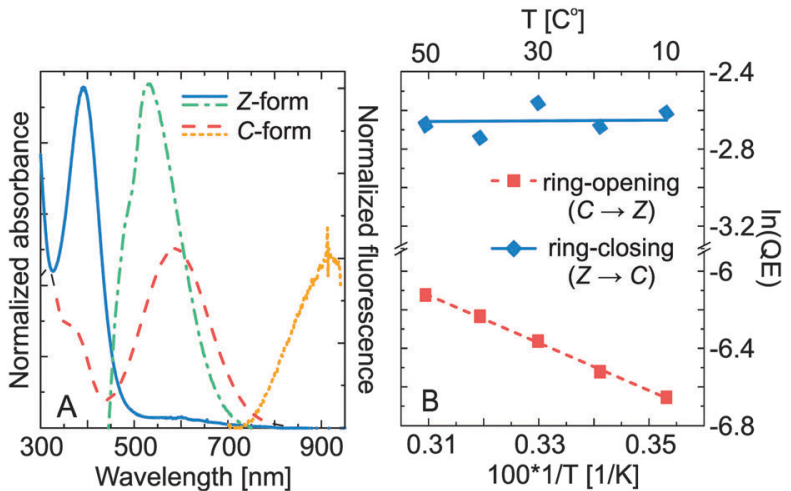

Fig. 1 (A) WF Z- and C-form absorption (solid blue and dashed red lines correspondingly) and fluorescence spectra (green dot-dash and yellow dotted lines correspondingly) in sodium phosphate buffer (pH 7.4). Extinction coefficients: $\varepsilon_{C, 588}=5600 \mathrm{~L} \mathrm{~mol}^{-1} \mathrm{~cm}^{-1} ; \varepsilon_{Z, 389}=9600 \mathrm{~L} \mathrm{~mol}^{-1} \mathrm{~cm}^{-1.33}$ (B) Arrhenius plots of the quantum yield for the ring-opening and ringclosing reaction.

show excellent thermal stability and fatigue resistance in organic solvents. ${ }^{6,20,21}$ The absorption spectra of their open and closed forms extend into the visible spectral range making them suitable for biochemical and biotechnological applications. Nevertheless, the presence of a succinic anhydride ring in indolylfulgides causes rapid solvolytic degradation in aqueous environments. $^{22}$ It has been shown that indolylfulgimides, where the succinic anhydride ring is replaced by a succinimide ring, have significantly improved hydrolytic stability ${ }^{6}$ and certain application examples are available. ${ }^{23,24}$

The ultrafast photochemical reactions of fulgides and fulgimides are well characterized in organic solvents. The ring-opening proceeds on the picosecond timescale ${ }^{25-28}$ after overcoming a barrier on the $S_{1}$ excited state potential energy surface. ${ }^{28,29}$ This makes the reaction sensitive to external factors like temperature, excitation wavelength and solvent polarity. The ring-closing reaction is barrierless (no dependence on the experimental conditions $)^{28,30}$ and typically occurs on the sub-picosecond timescale. ${ }^{31,32}$ Both reactions occur without the involvement of long-lived intermediates, which is an advantage over similar systems (e.g. spiropyrans).

Recently, we have reported on the synthesis of a watersoluble dicarboxylic acid indolylfulgimide (WF) (Scheme 1) suitable for applications in aqueous environments. ${ }^{33} \mathrm{WF}$ is thermally very stable in water (phosphate buffer, $\mathrm{pH}$ 7.4); after more than 20 days at $37{ }^{\circ} \mathrm{C}$ the compound degrades by less than $20 \%{ }^{33}$ Furthermore, the photochemical stability is also very high for a water-soluble photoswitch. WF is capable of 670 photochemical cycles before reaching $20 \%$ degradation. ${ }^{33}$ For comparison, the recently investigated water-soluble Py-BIPS compound shows $20 \%$ degradation in about 35 cycles. ${ }^{13}$ At present, there are no ultrafast studies on water-soluble fulgides or fulgimides and thus virtually nothing is known about the early photochemistry of those compounds in aqueous solutions. The information on the early photoreaction of fulgides and fulgimides is crucial for the intelligent design of new and improved compounds for extensive application in aqueous environments.
In this respect, we have focused our study on the investigation of the ultrafast ring-opening and ring-closing reactions of $\mathrm{WF}$ (Scheme 1).

\section{Materials and methods}

\section{Sample preparation}

The synthesis of the investigated water soluble dicarboxylic acid indolylfulgimide, has been previously described in detail. ${ }^{33}$ The compound in pure $C$-form was dissolved for the experiments in $50 \mathrm{mM}$ sodium phosphate buffer ( $\mathrm{pH} 7.4)$ or in $50 \mathrm{mM}$ sodium carbonate buffer $(\mathrm{pH}$ 9.8). The ratio of the different forms, $C: Z: E$, in the photostationary state (PSS) after $405 \mathrm{~nm}$ illumination as determined by ${ }^{1} \mathrm{H}$ NMR is $87: 6: 6 .^{33}$

\section{Steady-state spectroscopy and quantum yield determination}

The absorption spectra were recorded on a Specord S600 (Analytik Jena) absorption spectrometer using a fused silica cuvette with $1 \mathrm{~cm}$ optical path length. The temperature dependence $\left(10-50{ }^{\circ} \mathrm{C}\right)$ of the quantum yields (QY) for the ring-opening and -closing reactions were determined as described previously in details. ${ }^{28}$ High power LEDs (ThorLabs, M385L2, M530L2, driver DC4100) were used for the illumination of the $Z$ - and the $C$-forms. The intensity of the illuminating light was determined using a calibrated light detector (P-9710, Gigahertz-Optik). The PSS ${ }^{530}$ (530 $\mathrm{nm}$ illumination) and the PSS $^{385}$ (385 $\mathrm{nm}$ illumination) were always prepared at $20{ }^{\circ} \mathrm{C}$ before transferring the sample to the temperature of interest. The samples were continuously stirred using a magnetic stirrer (Thermo Scientific).

The fluorescence spectrum of the WF $Z$-form was recorded on a FP-8500 fluorimeter (Jasco Analytical Instruments, Tokyo) using a fused silica cuvette with $1 \mathrm{~cm}$ optical path length. The fluorescence spectrum of the WF $C$-form was recorded using a home-built CCD fluorimeter with sensitivity extending up to $\sim 950 \mathrm{~nm}$ using a fused silica with $1 \mathrm{~mm}$ optical path length. The fluorescence spectra were corrected for detector sensitivity and self-absorption.

The mid-IR absorption spectra of WF were recorded on a Vertex 80 FTIR-Spectrometer (Bruker, Ettlingen) using $50 \mu \mathrm{m}$ cuvettes built out of two $\mathrm{CaF}_{2}$ windows. The $C \rightarrow Z\left(\mathrm{PSS}^{\mathrm{VIS}}\right)$ and the $Z \rightarrow C\left(\mathrm{PSS}^{\mathrm{UV}}\right)$ conversions were accomplished by illuminating the samples directly in the spectrometer. The PSS ${ }^{\mathrm{VIS}}$ was reached by illumination with > $520 \mathrm{~nm}$ (HgXe arc lamp, Hamamatsu L958804, and a colour glass filter OG520, Schott AG), while the PSS ${ }^{\mathrm{UV}}$ was reached by illumination with $385 \mathrm{~nm}$ (LED, ThorLabs, M385L2).

\section{Sample conditions for the time-resolved experiments}

The $Z$ - and the $C$-form of the dicarboxylic acid indolylfulgimide were prepared at an OD of $\sim 0.7-1$. Fused silica cuvettes were used for the time-resolved experiments. For the $Z$-form a flowthrough cuvette was used ( 0.5 or $1 \mathrm{~mm}$ optical path length), while for the $C$-form a normal cuvette $(1 \mathrm{~mm}$ optical path length) that was continuously moved in the plane perpendicular to the direction of the probe pulse propagation was used. The samples were continuously illuminated to keep the PSS ${ }^{\mathrm{VIS}}$ 
( $>520 \mathrm{~nm}, \mathrm{HgXe}$ arc lamp, Hamamatsu L9588-04, and a colour glass filter OG520, Schott AG) or the PSS ${ }^{\mathrm{UV}}$ (385 nm, LED, ThorLabs, M385L2).

\section{UV-VIS pump-probe spectroscopy}

The femtosecond pump-probe setup used for the transient absorption measurements was described in detail previously. ${ }^{28}$ In short, the set-up is based on an oscillator-amplifier system (Clark, MXRCPA-iSeries, 1 kHz, 775 nm, 150 fs). The 590 nm pump pulses were generated in a two stage NOPA (non-collinear optical parametric amplifier) ${ }^{34,35}$ and compressed by a prism compressor to $\sim 40-50$ fs (determined with a PulseCheck USB 15 ShortPulse Autocorrelator, APE). The $390 \mathrm{~nm}$ pump pulses (150 fs) were produced from the laser fundamental $(775 \mathrm{~nm})$ by second harmonic generation. The pulse energy was adjusted to ensure that $<10 \%$ of the molecules are excited per pulse. Single filament white light (WL) pulses (300-700 nm) were generated by focusing the laser fundamental in a $5 \mathrm{~mm}$ thick $\mathrm{CaF}_{2}$ crystal. The detection of the transient signals was performed in referenced mode using the spectrometers described previously. ${ }^{28}$ The experiments were performed under magic angle conditions $\left(54.7^{\circ}\right.$ pump-probe polarization angle difference) to eliminate anisotropic contributions.

\section{Time-resolved fluorescence}

The femtosecond fluorescence experiments were conducted on a Kerr shutter set-up ${ }^{36,37}$ based on an oscillator-amplifier system (Tsunami-Spitfire Pro F, Spectra Physics, 1 kHz, 800 nm, $100 \mathrm{fs}$ ). The $400 \mathrm{~nm}$ excitation pulses for the $Z$-form were generated from the laser fundamental via second harmonic generation. The Kerr gate pulses $(1300 \mathrm{~nm})$ were generated using a home-built two stage OPA. ${ }^{38}$ The fluorescence was detected using a Spectrograph (Acton Research, SpektraPro 2358) and a CCD camera (Princeton Instruments, Spec-10:400B/LN). The wavelengthdependent time zero dispersion was corrected by using the Sellmeier equation. ${ }^{39,40}$

\section{Data analysis}

Global lifetime analysis (GLA) ${ }^{41,42}$ was used to analyse the fs transient absorption and time-resolved fluorescence data. In GLA the transients at all detection wavelengths are analysed simultaneously with a single set of exponential functions. The coherent artifact contribution at time zero in the transient absorption data was approximated with a function composed of a Gaussian and/or its first and second derivative ${ }^{43}$ and fitted within the same routine as the GLA. ${ }^{42}$ The data analysis was performed using OPTIMUS. ${ }^{42}$

\section{Theoretical calculations}

The theoretical calculations were performed with the help of Gaussian $09^{44}$ using density functional theory (DFT) and timedependent density functional theory (TD-DFT) ${ }^{45-47}$ in combination with the BHandHLYP functional ${ }^{48}$ and the $6-311 \mathrm{G}^{*}$ basis set. $^{49,50}$ Additionally, a polarizable continuum model (PCM) ${ }^{51}$ for water was applied and four explicit water molecules located in proximity to the carboxylic groups of the molecule were used. Due to the experimental conditions (phosphate buffer, $\mathrm{pH}$ 7.4) both carboxylic acid groups are deprotonated and therefore the deprotonated molecule was treated as doubly negatively charged anion.

\section{Results and discussion}

\section{Stationary spectroscopy and QY determination}

The absorption maximum of the $C$-form of WF dissolved in sodium phosphate buffer ( $\mathrm{pH} 7.4$ ) is located at $588 \mathrm{~nm}$, while the maximum of the $Z$-form is at $389 \mathrm{~nm}$ (Fig. 1). The position of the absorption bands of indolylfulgimides in different solvents (nonpolar or polar) depends strongly on the type of the substituents present in the molecule. ${ }^{33,52,53}$ Nevertheless, a clear solvent dependence can be observed for the absorption of the $C$-form which shifts strongly to the red side of the spectrum with increasing solvent polarity.

The QY of the ring-opening $(C \rightarrow Z)$ and the ring-closing $(Z \rightarrow C)$ reactions were measured as a function of temperature. The QY of the ring-closing reaction is $\sim 7 \%$ and is essentially temperature independent (Fig. 1B). Thus, similarly to other fulgides studied in organic solvents, ${ }^{28,30}$ in aqueous environment this reaction also proceeds without a barrier on the $S_{1}$ potential energy surface. In contrast, the QY of the ring-opening reaction shows a strong temperature dependence, going from $0.055 \%$ at $10{ }^{\circ} \mathrm{C}$ to $0.29 \%$ at $50{ }^{\circ} \mathrm{C}$ (Fig. 1B). Using the Arrhenius plots of the QY (Fig. 1B) and assuming $k_{\text {non-reactive }} \gg k_{\text {reactive, }}{ }^{54}$ the activation energy, $E_{\mathrm{a}}$, of the ring-opening reaction of $\mathrm{WF}$ in water was calculated to be $\sim 860 \mathrm{~cm}^{-1}\left(\sim 10 \mathrm{~kJ} \mathrm{~mol}^{-1}\right)$. This $E_{\mathrm{a}}$ is significantly larger than previously estimated for an indolylfulgimide in toluene and acetonitrile. ${ }^{53}$ Overall, the QY of both photochromic reactions of WF are strongly reduced as compared to indolylfulgimides studied in organic solvents. ${ }^{53,55,56}$ An additional QY measurement at higher $\mathrm{pH}$ shows that the ring-opening reaction is sensitive to the proton concentration in the solvent (at $20{ }^{\circ} \mathrm{C}: \mathrm{QY}_{\mathrm{pH}} 7.4=0.15 \% \mathrm{QY}_{\mathrm{pH} 9.8}=0.20 \%$ ), which indicates that the pathway of the ring-opening reaction of WF is affected by the protic nature of the solvent.

The photoinduced interconversion of WF $C$ - and $Z$-forms could also be monitored via steady-state FTIR spectroscopy (Fig. 2), which gives detailed insight into the rearrangement of the molecular structure. Previously, we have investigated a similar indolylfulgimide in a non-polar solvent and assigned the IR bands to specific vibrational modes of the molecule. ${ }^{26}$ Despite the different solvent used here and the differences in the substitution pattern, the majority of the IR bands of WF are preserved with a slight frequency shift. Additionally, we performed theoretical calculations using a polarizable continuum model for water and taking into account that under the experimental conditions used here (phosphate buffer, pD 7.4) the two carboxylic groups $\left(\mathrm{p} K_{\mathrm{a}}\right.$ typically $\left.<5\right)$ of $\mathrm{WF}$ are deprotonated. The model included also 4 explicit water molecules positioned in the vicinity of the carboxylic groups (ESI, $\dagger$ Fig. S1). The calculations are in excellent agreement with the experimental data and confirmed the former assignment of the IR bands (Fig. S2, ESI $\dagger$ ). 


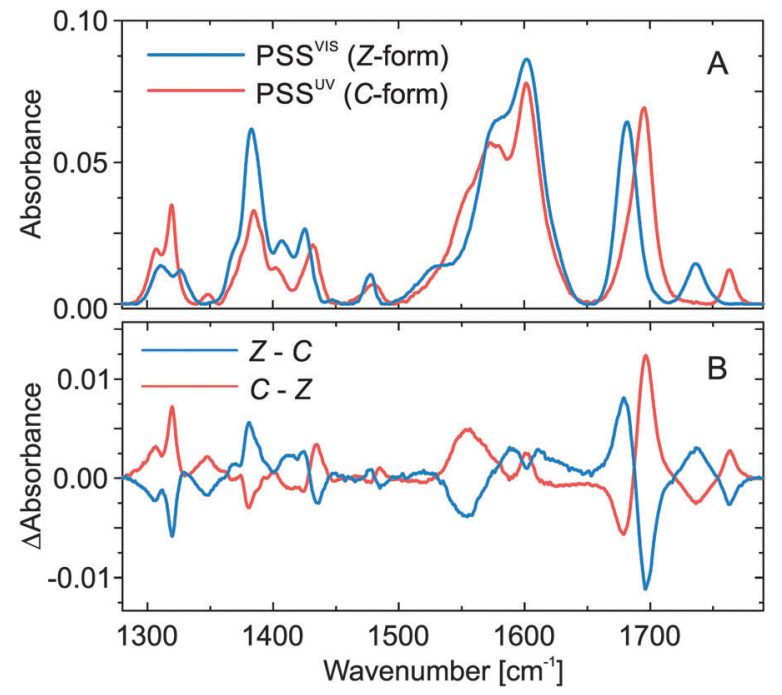

Fig. 2 (A) Infrared absorption spectra of the WF C- and Z-forms dissolved in sodium phosphate buffer ( $\mathrm{pD} 7.4$ ); (B) infrared absorption difference spectra resulting from the ring-opening and-closing reaction.

In the $C$-form, the frequencies of the asymmetric and the symmetric stretch modes of the CO groups from the succinimide ring are located at $1696 \mathrm{~cm}^{-1}$ and $1763 \mathrm{~cm}^{-1}$, respectively (Fig. 2). As a result of the $C \rightarrow Z$ ring-opening reaction, which changes the electronic configuration of the succinimide ring, the CO oscillation frequencies shift significantly down to $1682 \mathrm{~cm}^{-1}$ (asymmetric stretch) and $1736 \mathrm{~cm}^{-1}$ (symmetric stretch). In the absolute IR spectra (Fig. 2A) a strong and broad band is observed in the 1550-1650 $\mathrm{cm}^{-1}$ region, which does not change considerably during the $C \rightarrow Z$ conversion. However, in the difference spectra (Fig. 2B) a clear feature appears at $\sim 1550 \mathrm{~cm}^{-1}$. Based on the theoretical calculations the $1550 \mathrm{~cm}^{-1}$ band in the difference spectra (Fig. 2B and Fig. S2, ESI $\dagger$ ) was assigned to the $\mathrm{C}=\mathrm{C}$ stretching vibrations of the ring involved in the electrocyclic reaction, while the strong IR absorption band (1550-1650 $\left.\mathrm{cm}^{-1}\right)$ in the absolute spectra (Fig. 2A) was assigned to the asymmetric stretch of the $-\mathrm{COO}^{-}$groups. The rest of the pronounced bands $\left(<1450 \mathrm{~cm}^{-1}\right)$ are mostly due to CC (single and double bond) or NC (single bond) stretching modes from the different rings.

\section{Ultrafast ring-opening reaction $(C \rightarrow Z)$}

The ultrafast ring-opening reaction of the water-soluble indolylfulgimide (WF) was investigated using transient absorption spectroscopy with $\sim 50$ fs time resolution (Fig. 3A). The $C \rightarrow Z$ reaction was induced by excitation at the absorption maximum $(590 \mathrm{~nm})$ of the $C$-form. The transient absorption data is dominated by the presence of strong excited state absorption (ESA) signal due to the $\mathrm{S}_{1} \rightarrow \mathrm{S}_{n}$ transitions covering the complete detection window (Fig. 3A). In the range above $500 \mathrm{~nm}$ the ESA overlaps with the ground state bleach signal (GSB). The GSB has the same spectral shape as the $C$-form absorption spectrum (Fig. 1A, maximum at $\sim 590 \mathrm{~nm}$ ) but with negative sign and thus partially compensates the ESA in this spectral range. This compensation causes a reduced amplitude in the ESA spectrum around $590 \mathrm{~nm}$. An additional ESA band,

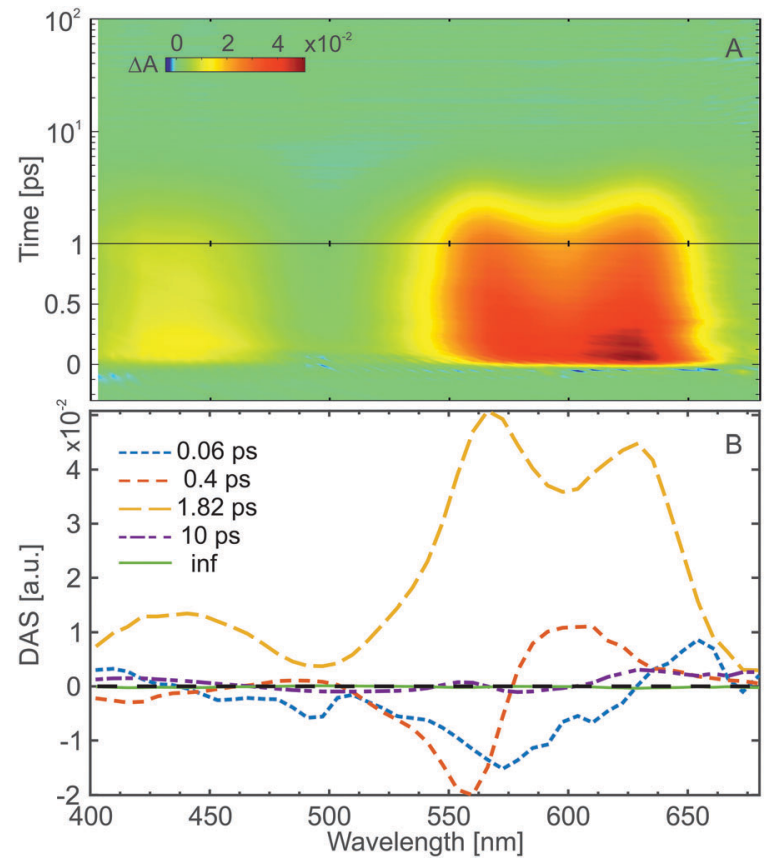

Fig. 3 Results from the investigation of the $C \rightarrow Z$ ring-opening reaction of WF dissolved in sodium phosphate buffer ( $\mathrm{pH}$ 7.4). (A) Transient absorption data after $590 \mathrm{~nm}$ excitation. (B) Decay-associated spectra (DAS) obtained from the global analysis (see Materials and methods) of the transient absorption data.

associated with another $S_{1} \rightarrow S_{n}$ transition, is present below $470 \mathrm{~nm}$.

The decay-associated spectra (DAS) resulting from the global lifetime analysis (GLA $)^{41,42}$ is composed of four lifetimes and a non-decay component (inf) (Fig. 3B). The $0.06 \mathrm{ps}$ and the $0.4 \mathrm{ps}$ components show typical positive $(600-650 \mathrm{~nm})$ - negative (550-570 nm) amplitude features, which are due to the shift of the corresponding band (in this case ESA around $600 \mathrm{~nm}$ ) to higher energies. In this respect, those two lifetime components in the ultrafast kinetics of the $C$-form can be assigned to the relaxation of the excited molecule from the Franck-Condon region and to solvent reorganization. The dominant third lifetime component (1.8 ps) is characterized by an all-positive DAS, reflecting the decay of the ESA bands and the repopulation of the ground state. The fourth lifetime component $(\sim 10 \mathrm{ps})$, associated with the vibrational cooling of the ground state, shows only a minor amplitude contribution. The non-decaying component (inf) corresponds to the final absorption difference spectrum. This spectrum is nearly flat for the $C$-form, which reflects the low amount of product formation. This result is explained by the relatively low QY of the ring-opening reaction $\left(\sim 0.15 \%\right.$ at $\left.23{ }^{\circ} \mathrm{C}\right)$, which does not permit the detection of the product formation in the transient absorption experiment.

Overall, the ultrafast $C \rightarrow Z$ ring-opening reaction of WF in water shows similar dynamics to previously studied indolylfulgimides. Nevertheless, an important solvent effect arises from the comparison with the previous studies. While the change from non-polar (tetrachloroethylene or toluene) ${ }^{27,53}$ to polar aprotic solvent (acetonitrile) ${ }^{31,35,41}$ leads to an increase of the 
excited state lifetime of the $C$-form ( 2 ps $\rightarrow 3$ ps), an opposite trend is detected in water (polar protic solvent) where the excited state lifetime (1.8 ps) becomes even shorter than in non-polar solvents. Considering the significantly lower ring-opening QY in water and the increased energy barrier along the reaction pathway, the shortening of the excited state lifetime could be attributed to an increased preference of the internal conversion pathway. Our theoretical calculations for the relaxed $S_{0}$ and $S_{1}$ structures of the $C$-form showed that the dipole moment of the molecule in the excited state $(\sim 33.5 \mathrm{D})$ is larger than in the ground state $(\sim 26.2 \mathrm{D})$. Hence, the excited state of the $C$-form is stabilized in polar solvents. However, the short excited state lifetime observed in the present experiments with WF indicates that due to the protic nature of water an additional relaxation pathway may play a role. Previous theoretical calculations indicated that the excited state dynamics of the $C$-form is governed by the interplay of two conical intersections, where only one has a contribution to the ring-opening reaction, while the other one leads to fast deactivation into the $C$-form ground state. ${ }^{57,58}$ Within this conical intersection picture the shortened excited state lifetime of the $C$-form in protic solvents could be caused either by the opening of a new proton transfer reaction channel or an improved accessibility of the conical intersection leading to the $C$-form ground state.

We investigated the changes in the partial charge distributions in the $C$-form of the WF molecule occurring after the transition to the excited state to identify potential positions for proton transfer reactions (Table S1, ESI $\dagger$ ). Interestingly, the typical candidate for excited state proton transfer reactions (the $\mathrm{O}$ atoms) showed only minor photoinduced changes in their partial charges. The largest effects were observed for the $\mathrm{N}$ atoms of the indolyl group and the succinimide ring (positions 9 and 15) and the $\mathrm{C}$ atoms of the photochromic ring III (positions 8, 10, and 11) and at the succinimide ring (position 16) (Fig. S3, ESI $\dagger$ ).

Coherent oscillations in the ultrafast transient absorption signals of the $C$-form of indolylfulgides ${ }^{28,30}$ and indolylfulgimides ${ }^{53}$ have been detected previously. Such oscillations are present also in the transient absorption data from WF in water (clearly visible in the red edge of the ESA, Fig. 3A and in the single transients, Fig. 4A). The Fourier analysis of the oscillatory pattern shows that the dominant frequency is $\sim 80 \mathrm{~cm}^{-1}$ (Fig. $4 \mathrm{~B}$ ). Several other higher frequencies could also be resolved $\left(\sim 170, \sim 290, \sim 410, \sim 530 \mathrm{~cm}^{-1}\right)$. Recently, for an indolylfulgide, we have showed that the observed coherent oscillation $\left(\sim 80 \mathrm{~cm}^{-1}\right)$ is linked to vibrational wavepacket motion on the excited state potential energy surface. ${ }^{28}$ The theoretical analysis for this indolylfulgide indicated that the motion is due to a vibrational mode contributing significantly to the ring-opening reaction coordinates. ${ }^{28}$ The same dominant frequency appears here also for the WF. Consequently, this vibrational mode is universally involved in the reaction dynamics of the $C$-form of fulgides.

\section{Ultrafast ring-closing reaction $(Z \rightarrow C)$}

Transient absorption (Fig. 5A, $390 \mathrm{~nm}$ excitation) as well as timeresolved fluorescence spectroscopy (Fig. 5C, $400 \mathrm{~nm}$ excitation) were employed to investigate the ultrafast ring-closing reaction of the water-soluble fulgimides (WF). The excitation wavelength in both experiments was tuned near the absorption maximum of the

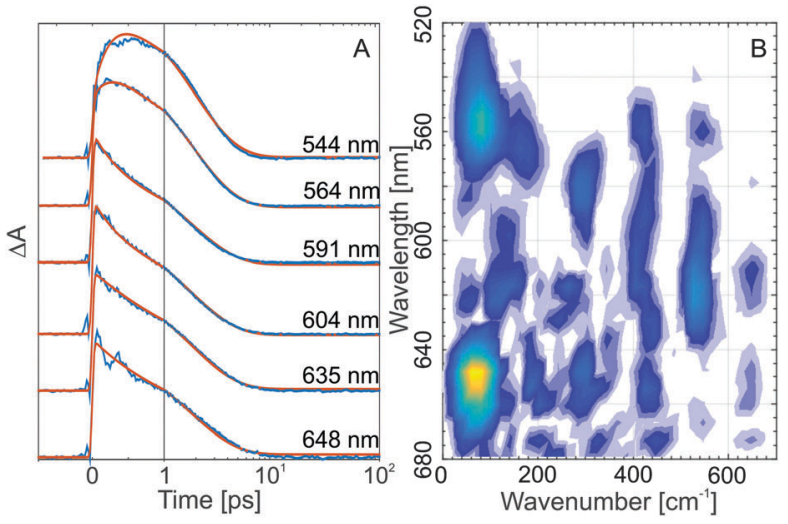

Fig. 4 (A) Coherent oscillations in the single absorption difference transients recorded after excitation $(590 \mathrm{~nm})$ of the $C$-form of WF in sodium phosphate buffer ( $\mathrm{pH}$ 7.4). (B) Frequency map obtained from the Fourier analysis of the residuals from the multi-exponential fit of the transient absorption data.

open $Z$-form (see Materials and methods for the Experimental details). The transient absorption data (Fig. 5A) is dominated by the strong GSB of the $Z$-form $(\sim 400 \mathrm{~nm})$, as well as by two strong ESA bands $(<370 \mathrm{~nm}$ and $>440 \mathrm{~nm})$. In addition, on the early timescale $(<500 \mathrm{fs})$ and above $570 \mathrm{~nm}$, also stimulated emission (SE) contribution is discernible. The formation of the $C$-form product as a result of the $Z$-form photoinduced cyclization is

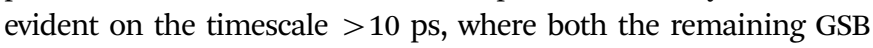
band $(\sim 400 \mathrm{~nm})$ due to the non-recovered $Z$-form, as well as the newly formed $C$-form absorption band $(\sim 590 \mathrm{~nm})$ are present.

The GLA of the $Z$-form transient absorption data yielded three lifetimes and a non-decaying component (inf) (Fig. 5B). The shortest lifetime component $(0.14 \mathrm{ps})$ is associated with the relaxation of the excited $Z$-form from the Franck-Condon region and towards the conical intersection to the ground state. The DAS of this lifetime is a complex mixture reflecting spectral shifts of the ESA and the SE bands. The main decay of the ESA and recovery of the GSB occurs with $0.4 \mathrm{ps}$ lifetime. With this lifetime, also the rise of the $C$-form product absorption can be observed. Relaxation of the excited state is followed by vibrational cooling in the ground state ( $3.3 \mathrm{ps}$ lifetime). The hot ground state band (broad absorption peaking at $450 \mathrm{~nm}$ ) shifts with the $3.3 \mathrm{ps}$ lifetime to $400 \mathrm{~nm}$ to partially repopulate the relaxed ground state and further reveals on the red side of the spectral range $(>500 \mathrm{~nm}$ ) the absorption of the $C$-form product.

The assignment of the dynamics in the transient absorption data is confirmed by time-resolved fluorescence (Fig. 5C). The spectrally very broad and ultrashort fluorescence has an initial maximum at $\sim 530 \mathrm{~nm}$, which quickly shifts to the red and decays on the timescale $<2$ ps. The spectral properties of the $Z$-form time-resolved fluorescence match the stationary fluorescence spectrum (Fig. 1A). Two lifetimes were sufficient to fit the time-resolved fluorescence of the $Z$-form. The first one, $\sim 100 \mathrm{fs}$, accounts for the ultrafast red-shift of the fluorescence due to the departure of the excited $Z$-form from the Franck-Condon region. The second one, $\sim 400 \mathrm{fs}$, represents the decay of the fluorescence and thus the decay of the excited state. Therefore, the conclusion drawn from the time-resolved fluorescence and 

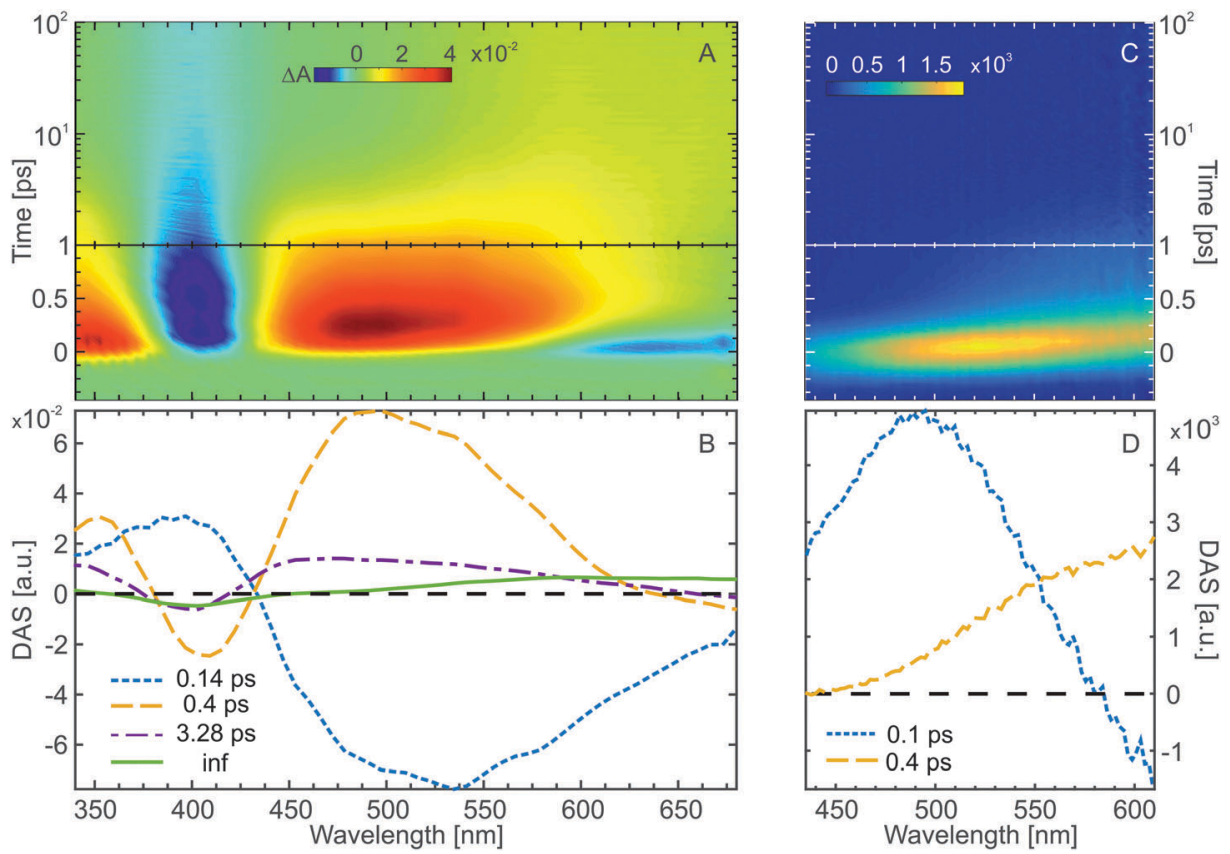

Fig. 5 Results from the investigation of the $Z \rightarrow C$ ring-closing reaction of WF dissolved in sodium phosphate buffer (pH 7.4). (A) Transient absorption data after excitation at $390 \mathrm{~nm}$. (B) DAS obtained from the GLA of the transient absorption data. (C) Time-resolved fluorescence data after excitation at $400 \mathrm{~nm}$. (D) DAS obtained from the GLA of the time-resolved fluorescence data.

the transient absorption data of the $Z$-form are in excellent agreement. Furthermore, the excited state reaction dynamics of the $Z$-form of the water-soluble indolylfulgimide is very similar to what was previously observed for a related compound dissolved in acetonitrile. ${ }^{31,59}$ The main difference for those two compounds and solvents is found in the vibrational cooling lifetime, which is nearly two times faster for WF.

\section{Conclusions}

In the current work, we present the first, to our knowledge, investigation of the ultrafast photoconversion dynamics of a water-soluble fulgimide (WF). Despite the reduced QY of this indolylfulgimide in water, as compared to similar compounds dissolved in organic solvents, WF shows very high thermal- and photostability. The kinetics of the ring-closing $Z \rightarrow C$ reaction is mostly similar to the kinetics of indolylfulgimides in polar solvents. The conical intersection to the ground state for this reaction is readily accessible as there is no energetic barrier on the relaxation pathway from the Franck-Condon region (Scheme 2). Thus the reaction is generally ultrafast $(\sim 0.4 \mathrm{ps})$ and is not affected significantly by changes in the environment. The $C \rightarrow Z$ ring-opening reaction in water, on the other hand, is faster than in both polar and non-polar organic solvents. Surprisingly, this is an opposite trend as typically the reaction is slowed down in polar solvents due to stabilization of the polar excited state. Evidently, in protic solvents like water, excited state proton transfer reactions play a role in the relaxation kinetics of photoexcited fulgimides. Such reactions effectively quench the excited state, which reduces the QY of the photoconversion. In this respect, a major solvent effect on the ultrafast kinetics

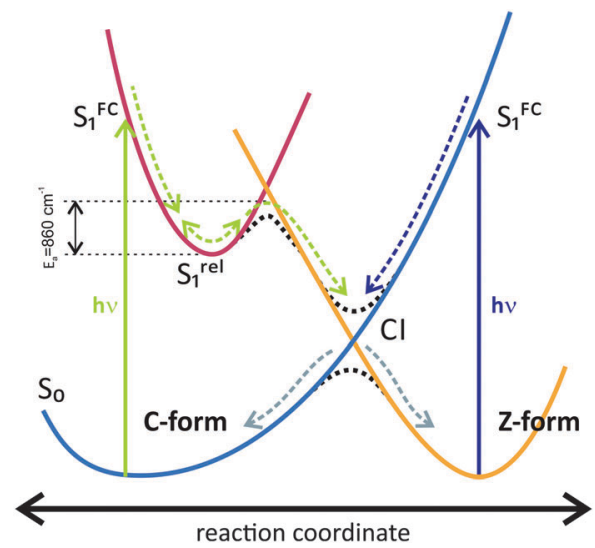

Scheme 2 Reaction scheme for the ring-opening and-closing reactions of the water-soluble WF.

of indolylfulgimides emerges from our study, which should serve as a basis for future optimizations of the WF structure.

\section{Acknowledgements}

Chavdar Slavov and Josef Wachtveitl acknowledge DFG (Grant WA 1850/4-1).

\section{References}

1 G. H. Brown, Photochromism, Wiley, New York, 1971.

2 Photochromism: Molecules and Systems, ed. H. Dürr and H. Bouas-Laurent, Elsevier, Amsterdam, 2003.

3 N. Tamai and H. Miyasaka, Chem. Rev., 2000, 100, 1875-1890. 
4 R. H. El Halabieh, O. Mermut and C. J. Barrett, Pure Appl. Chem., 2004, 76, 1445-1465.

5 M. Irie, Chem. Rev., 2000, 100, 1685-1716.

6 Y. Yokoyama, Chem. Rev., 2000, 100, 1717-1739.

7 R. Klajn, Chem. Soc. Rev., 2014, 43, 148-184.

8 J. Garcia-Amorós, M. Díaz-Lobo, S. Nonell and D. Velasco, Angew. Chem., Int. Ed., 2012, 51, 12820-12823.

9 A. A. Beharry, O. Sadovski and G. A. Woolley, J. Am. Chem. Soc., 2011, 133, 19684-19687.

10 R. Matsushima and H. Sakaguchi, J. Photochem. Photobiol., A, 1997, 108, 239-245.

11 T. Stafforst and D. Hilvert, Chem. Commun., 2009, 287-288.

12 X. Chen, N. I. Islamova, R. V. Robles and W. J. Lees, Photochem. Photobiol. Sci., 2011, 10, 1023-1029.

13 J. Kohl-Landgraf, M. Braun, C. Özçoban, D. P. N. Gonçalves, A. Heckel and J. Wachtveitl, J. Am. Chem. Soc., 2012, 134, 14070-14077.

14 O. Sadovski, A. A. Beharry, F. Z. Zhang and G. A. Woolley, Angew. Chem., Int. Ed., 2009, 48, 1484-1486.

15 M. A. Cardona and D. C. Magri, Tetrahedron Lett., 2014, 55, 4559-4563.

16 C. Özçoban, T. Halbritter, S. Steinwand, L.-M. Herzig, J. KohlLandgraf, N. Askari, F. Groher, B. Fürtig, C. Richter, H. Schwalbe, B. Suess, J. Wachtveitl and A. Heckel, Org. Lett., 2015, 17, 1517-1520.

17 S. Heng, C. A. McDevitt, D. B. Stubing, J. J. Whittall, J. G. Thompson, T. K. Engler, A. D. Abell and T. M. Monro, Biomacromolecules, 2013, 14, 3376-3379.

18 S. Steinwand, T. Halbritter, D. Rastädter, J. M. Ortiz-Sánchez, I. Burghardt, A. Heckel and J. Wachtveitl, Chem. - Eur. J., 2015, 21, 15720-15731.

19 F. Renth, R. Siewertsen and F. Temps, Int. Rev. Phys. Chem., 2012, 32, 1-38.

20 Y. Yokoyama and K. Takahashi, Chem. Lett., 1996, 1037-1038.

21 N. I. Islamova, X. Chen, S. P. Garcia, G. Guez, Y. Silva and W. J. Lees, J. Photochem. Photobiol., A, 2008, 195, 228-234.

22 A. Kaneko, A. Tomoda, M. Ishizuka, H. Suzuki and R. Matsushima, Bull. Chem. Soc. Jpn., 1988, 61, 3569-3573.

23 M. W. Berns, T. Krasieva, C. H. Sun, A. Dvornikov and P. M. Rentzepis, J. Photochem. Photobiol., B, 2004, 75, 51-56.

24 F. D. Jochum, F. R. Forst and P. Theato, Macromol. Rapid Commun., 2010, 31, 1456-1461.

25 H. Port, P. Gärtner, M. Hennrich, I. Ramsteiner and T. Schock, Mol. Cryst. Liq. Cryst., 2005, 430, 15-21.

26 F. O. Koller, W. J. Schreier, T. E. Schrader, A. Sieg, S. Malkmus, C. Schulz, S. Dietrich, K. Rück-Braun, W. Zinth and M. Braun, J. Phys. Chem. A, 2006, 110, 12769-12776.

27 S. Malkmus, F. O. Koller, B. Heinz, W. J. Schreier, T. E. Schrader, W. Zinth, C. Schulz, S. Dietrich, K. RückBraun and M. Braun, Chem. Phys. Lett., 2006, 417, 266-271.

28 C. Slavov, N. Bellakbil, J. Wahl, K. Mayer, K. Rück-Braun, I. Burghardt, J. Wachtveitl and M. Braun, Phys. Chem. Chem. Phys., 2015, 17, 14045-14053.

29 S. Draxler, T. Brust, S. Malkmus, J. A. DiGirolamo, W. J. Lees, W. Zinth and M. Braun, Phys. Chem. Chem. Phys., 2009, 11, 5019-5027.
30 T. Brust, S. Draxler, A. Popp, X. Chen, W. J. Lees, W. Zinth and M. Braun, Chem. Phys. Lett., 2009, 477, 298-303.

31 B. Heinz, S. Malkmus, S. Laimgruber, S. Dietrich, C. Schulz, K. Rück-Braun, M. Braun, W. Zinth and P. Gilch, J. Am. Chem. Soc., 2007, 129, 8577-8584.

32 M. Handschuh, M. Seibold, H. Port and H. C. Wolf, J. Phys. Chem. A, 1997, 101, 502-506.

33 X. Chen, N. I. Islamova, S. P. Garcia, J. A. DiGirolamo and W. J. Lees, J. Org. Chem., 2009, 74, 6777-6783.

34 T. Wilhelm, J. Piel and E. Riedle, Opt. Lett., 1997, 22, 1494-1496.

35 E. Riedle, M. Beutter, S. Lochbrunner, J. Piel, S. Schenkl, S. Spörlein and W. Zinth, Appl. Phys. B: Lasers Opt., 2000, 71, 457-465.

36 B. Schmidt, S. Laimgruber, W. Zinth and P. Gilch, Appl. Phys. B: Lasers Opt., 2003, 76, 809-814.

37 P. Trojanowski, PhD dissertation, Goethe University, 2015.

38 G. Cerullo and S. De Silvestri, Rev. Sci. Instrum., 2003, 74, 1-18.

39 W. Sellmeier, Ann. Phys., 1871, 219, 272-282.

40 D. Meschede, Optics, Light and Lasers, Wiley-VCH, 2007.

41 I. H. M. van Stokkum, D. S. Larsen and R. van Grondelle, Biochim. Biophys. Acta, Bioenerg., 2004, 1657, 82-104.

42 C. Slavov, H. Hartmann and J. Wachtveitl, Anal. Chem., 2015, 87, 2328-2336.

43 S. A. Kovalenko, A. L. Dobryakov, J. Ruthmann and N. P. Ernsting, Phys. Rev. A: At., Mol., Opt. Phys., 1999, 59, 2369-2384.

44 M. J. Frisch, G. W. Trucks, H. B. Schlegel, G. E. Scuseria, M. A. Robb, J. R. Cheeseman, G. Scalmani, V. Barone, B. Mennucci, G. A. Petersson, H. Nakatsuji, M. Caricato, X. Li, H. P. Hratchian, A. F. Izmaylov, J. Bloino, G. Zheng, J. L. Sonnenberg, M. Hada, M. Ehara, K. Toyota, R. Fukuda, J. Hasegawa, M. Ishida, T. Nakajima, Y. Honda, O. Kitao, H. Nakai, T. Vreven, J. A. Montgomery Jr., J. E. Peralta, F. Ogliaro, M. J. Bearpark, J. Heyd, E. N. Brothers, K. N. Kudin, V. N. Staroverov, R. Kobayashi, J. Normand, K. Raghavachari, A. P. Rendell, J. C. Burant, S. S. Iyengar, J. Tomasi, M. Cossi, N. Rega, N. J. Millam, M. Klene, J. E. Knox, J. B. Cross, V. Bakken, C. Adamo, J. Jaramillo, R. Gomperts, R. E. Stratmann, O. Yazyev, A. J. Austin, R. Cammi, C. Pomelli, J. W. Ochterski, R. L. Martin, K. Morokuma, V. G. Zakrzewski, G. A. Voth, P. Salvador, J. J. Dannenberg, S. Dapprich, A. D. Daniels, Ö. Farkas, J. B. Foresman, J. V. Ortiz, J. Cioslowski and D. J. Fox, Gaussian 09, Gaussian, Inc., Wallingford, CT, USA, 2009.

45 E. Runge and E. K. U. Gross, Phys. Rev. Lett., 1984, 52, 997-1000. 46 M. E. Casida, Recent Advances in Density Functional Methods, World Scientific, 1995.

47 A. Dreuw and M. Head-Gordon, Chem. Rev., 2005, 105, 4009-4037.

48 A. D. Becke, J. Chem. Phys., 1993, 98, 1372-1377.

49 R. Krishnan, J. S. Binkley, R. Seeger and J. A. Pople, J. Chem. Phys., 1980, 72, 650-654.

50 A. D. McLean and G. S. Chandler, J. Chem. Phys., 1980, 72, 5639-5648.

51 J. Tomasi, B. Mennucci and R. Cammi, Chem. Rev., 2005, 105, 2999-3094. 
52 N. I. Islamova, X. Chen, J. A. DiGirolamo, Y. Silva and W. J. Lees, J. Photochem. Photobiol., A, 2008, 199, 85-91.

53 T. Brust, S. Malkmus, S. Draxler, S. A. Ahmed, K. RückBraun, W. Zinth and M. Braun, J. Photochem. Photobiol., A, 2009, 207, 209-216.

54 T. Brust, S. Draxler, S. Malkmus, C. Schulz, M. Zastrow, K. Rück-Braun, W. Zinth and M. Braun, J. Mol. Liq., 2008, 141, 137-139.

55 F. O. Koller, W. J. Schreier, T. E. Schrader, S. Malkmus, C. Schulz, S. Dietrich, K. Rück-Braun and M. Braun, J. Phys. Chem. A, 2008, 112, 210-214.
56 M. A. Wolak, N. B. Gillespie, C. J. Thomas, R. R. Birge and W. J. Lees, J. Photochem. Photobiol., A, 2001, 144, 83-91.

57 D. Geppert, L. Seyfarth and R. de Vivie-Riedle, Appl. Phys. B: Lasers Opt., 2004, 79, 987-992.

58 A. Nenov, W. J. Schreier, F. O. Koller, M. Braun, R. de VivieRiedle, W. Zinth and I. Pugliesi, J. Phys. Chem. A, 2012, 116, 10518-10528.

59 S. Draxler, T. Brust, S. Malkmus, F. O. Koller, B. Heinz, S. Laimgruber, C. Schulz, S. Dietrich, K. Rück-Braun, W. Zinth and M. Braun, J. Mol. Liq., 2008, 141, 130-136. 